\title{
Vorwort der Herausgeber zur 2. Auflage
}

Auch zwei Jahre nach dem Erscheinen der ersten Auflage des vorliegenden Handbuchs ist das Recht der Bankenaufsicht hoch dynamischen Veränderungen unterworfen. Die mit dem Bankenpaket 2019 verabschiedeten Anpassungen und Ergänzungen sowohl des materiellen Aufsichtsrechts als auch des Rechtsrahmens für die Abwicklung systemrelevanter Banken befinden sich inzwischen im Implementierungsstadium. Schon sie rechtfertigen die vorliegende Neuauflage, nachdem die Erstauflage überaus freundlich aufgenommen wurde. Unverändert gilt: Die Aufarbeitung dieser Entwicklungen und deren praktische Umsetzung stellen die Aufsichts- und Beratungspraxis ebenso wie die Wissenschaft vor erhebliche Herausforderungen; der Beratungsbedarf ist groß und wächst weiterhin. Wie die erste Auflage des Handbuchs bemüht sich auch der vorliegende Band darum, die Materie, soweit geboten, interdisziplinär aufzubereiten und damit Praxis und Wissenschaft einen umfassenden Zugriff auf die für die Auslegung und Handhabung des Aufsichtsrechts relevanten Fragestellungen zu ermöglichen.

Auch die 2. Auflage des Handbuchs ist in puncto Aktualität notgedrungen von Kompromissen geprägt. In jüngster Zeit sind in Reaktion auf die Corona-Krise (nicht nur) innerhalb der Bankenunion grundlegende Erleichterungen im materiellen Aufsichtsrecht vorgenommen wurden, um das Bankenwesen vor den unmittelbaren Ertragsausfällen zu schützen und die Folgen der Krise für die Kreditvergabe abzumildern.*) Diese Entwicklungen trafen zeitlich mit der Fertigstellung des Manuskripts zur vorliegenden 2. Auflage zusammen. Vor die Wahl zwischen dem raschen Erscheinen und der Berücksichtigung dieser Entwicklungen im Text gestellt, haben sich Herausgeber und Verlag für ersteres entschieden - auch mit Blick auf die voraussichtlich lediglich temporäre Natur der Veränderungen. Auch weiterhin wird indessen eine regelmäßige Auflagenfolge angestrebt, um das Handbuch möglichst aktuell zu halten.

Der Dank der Herausgeber gilt zunächst dem engagierten Autorenteam für die zuverlässige und gründliche Aktualisierung der bereits vorhandenen Beiträge bzw. - den neu hinzugekommenen Autorinnen und Autoren - für fundierte neue Kapitel. Wiederum danken wir überdies dem RWS-Verlag und hier insbesondere Frau Iris Theves-Telyakar für das äußerst gewissenhafte Lektorat sowie dem Verlagsleiter, Herrn Markus Sanerwald, für die stetige Unterstützung des Buchprojekts. Zu danken ist außerdem den Mitarbeitern am Tübinger Lehrstuhl für Bürgerliches Recht, Handels- und Wirtschaftsrecht, insbesondere Frau Ingrid Hoch sowie Herrn cand. iur. Johannes Koch, die mit umsichtiger editorischer Betreuung der Manuskripte Wertvolles zum Gesamtwerk beigetragen haben.

Anregungen und Verbesserungsvorschläge - idealerweise per E-Mail (lehrstuhl.binder@ jura.uni-tuebingen.de) - sind auch künftig gerne willkommen.

Tübingen und Frankfurt am Main, im Mai 2020

Jens-Hinrich Binder

Alexander Glos

Jan Riepe

*) S. im Überblick https://www.bankingsupervision.europa.eu/home/search/coronavirus/html/index.en.html; https://ec.europa.eu/finance/docs/law/200428-banking-package-communication_en.pdf; https://eba. europa.eu/eba-provides-clarity-banks-consumers-application-prudential-framework-light-covid-19measures. 
